\title{
AVALIAÇÃO DE PROPRIEDADES FÍSICAS E MECÂNICAS DA MADEIRA DE CINCO ESPÉCIES FLORESTAIS EM FUNÇÃO DA DETERIORAÇÃO EM DOIS AMBIENTES ${ }^{1}$
}

\author{
Henrique Trevisan², Felipe Marauê Marques Tieppo ${ }^{3}$, Acacio Geraldo de Carvalho ${ }^{4}$ e Roberto Carlos \\ Costa Lelis ${ }^{4}$
}

\begin{abstract}
RESUMO - Este trabalho teve por objetivo avaliar algumas propriedades físicas e mecânicas da madeira de Eucalyptus urophylla S.T. Blake "Eucalipto" (Myrtaceae), Melia azedarach L. "Cinamomo" (Meliaceae), Lophantera lactescens Ducke, "lanterneira" (Malpighiaceae), Pinus elliottii Engelm. "Pinus" (Pinaceae) e Inga marginata Wild "Inga" (Mimosaceae), submetidas ao processo de degradação em razão da exposição à intempérie no período de 12 meses, as madeiras foram colocadas em dois ambientes com características edafoclimáticas diferenciadas, isto é, foram montados dois campos de apodrecimento, sendo um dentro de uma mata secundária e outro a céu aberto, em um pasto formado por gramíneas rasteiras. Na avaliação, adotouse um índice de deterioração médio, para expressar a degradação causada pelos fatores bióticos, nos corposde-prova oriundos de toras expostas nos respectivos ambientes. As determinações da densidade aparente, bem como da resistência à flexão (módulo de elasticidade (MOE) e ruptura (MOR)) e compressão paralela às fibras da madeira, foram realizadas antes e depois da exposição à intempérie. Os resultados indicaram que ocorreram reduções diferenciadas nas propriedades avaliadas. As diminuições significativas da densidade ocorreram somente nos corpos-de-prova oriundos das madeiras expostas dentro da mata, bem como as reduções nos módulos de elasticidade e ruptura foram mais acentuadas nas madeiras procedentes desse ambiente. A resistência à compressão da madeira apresentou-se com reduções maiores, também nesse ambiente.
\end{abstract}

Palavras-chave: Madeira, deterioração e propriedades tecnológicas.

\section{EVALUATION OF PHYSICAL AND MECHANICAL PROPERTIES OF WOOD FROM FIVE SPECIES AS A FUNCTION OF DETERIORATION IN TWO ENVIROMENTS}

\begin{abstract}
The objective of this work was to evaluate some physical and mechanical properties of Eucalyptus urophylla S.T. Blake - Eucalyptus (Myrtaceae), Melia azedarach L. - Chinaberry (Meliaceae), Lophantera lactescens Ducke - "Lanterneira" (Malpighiaceae), Pinus elliottii Engelm. - Slash Pine (Pinaceae), Inga marginata Wild - Inga (Mimosaceae), subjected to the degradation process caused by the exposition to atmospheric conditions over a period of 12 months, in two environments with different edafoclimatic characteristics, i.e., two decaying fields were set up, one within a secondary forest and the other in an open field, a pasture composed by low grass. For the evaluation, we adapted an average decay index, to express the degradation caused by biotic factors in specimens from wood exposed to both environments. The determination of apparent density,
\end{abstract}

\footnotetext{
${ }^{1}$ Recebido em 11.05.2006 e aceito para publicação em 20.10.2006.

${ }^{2}$ Programa de Pós-Graduação em Ciências Ambientais e Florestais da UFRRJ. E-mail: <hentrevisan@ gmail.com>.

${ }^{3}$ Graduação de Engenharia Florestal da UFRRJ.

${ }^{4}$ Departamento de Produtos Florestais da UFRRJ.
} 
as well as the resistance to beding in the modulus of elasticity (MOE) and rupture (MOR) and parallel compression to wood fiber, was carried out before and after the exposure to atmospheric conditions. The results showed the occurrence of different reductions in the evaluated properties. Significant reductions in density occurred only in the wood originated from inside the forest, as well as reductions in the Modulus of elasticity and rupture were more accentuated in wood originate from this environment. Resistance to compression has also shown major reductions in the same environment.

Keywords: Wood, deterioration and technological properties.

\section{INTRODUÇÃO}

Segundo Aprile et al. (1999), a madeira encontrase entre os materiais biológicos de mais difícil decomposição devido à sua estrutura anatômica e à presença de grandes quantidades de substâncias recalcitrantes como a lignina, além de outros compostos do metabolismo secundário. Sabe-se que a decomposição de materiais biológicos depende de uma série de fatores bióticos e abióticos, como as condições climáticas, a composição da comunidade decompositora, sua afinidade para com o substrato e as características físicas e químicas do material a ser decomposto (SWIFT et al., 1979).

Nesse sentido, o estudo da degradação da madeira deve envolver áreas distintas do conhecimento; entre elas podem ser citadas: a entomologia, micologia, fisiologia vegetal e a tecnologia da madeira, sendo de extrema importância o sinergismo entre elas, para que o processo de degradação seja compreendido de forma integral, gerando uma base de dados sobre o comportamento dos organismos xilófagos, seja nas interações ecológicas entre eles ou com a própria madeira estudada.

A tecnologia da madeira cumpre papel fundamental na elucidação da deterioração dese material, pois as informações sobre as propriedades físicas e mecânicas são oriundas dessa área. Portanto, com relação às propriedades mecânicas, o estudo desse parâmetro, em trabalhos que avaliam a decomposição da madeira, pode fornecer informações valiosas quanto à redução dessas características diante da ação do processo de degradação, aumentando, de forma valiosa, o banco de dados de cada espécie, no que se refere à ação da decomposição, interferindo nessas características.

A elucidação desses processos pode, assim, nortear o uso da madeira, pois se conhecerá o desempenho das propriedades tecnológicas intrínsecas a cada espécie florestal, diante do processo de ação dos organismos xilófagos e dos fatores abióticos, atuantes na deterioração da madeira.

O objetivo deste trabalho foi avaliar a densidade aparente e a resistência mecânica através do módulo de elasticidade (MOE) e ruptura (MOR) na flexão estática e da resistência à compressão paralela às fibras, de cinco madeiras, em função da atuação da deterioração, propiciada pela exposição, ao longo de 12 meses, a intempéries, em dois ambientes.

\section{MATERIAL E MÉTODOS}

A madeira utilizada neste trabalho foi obtida de árvores cultivadas no Campus da Universidade Federal Rural do Rio de Janeiro (UFRRJ) e na Floresta Nacional Mário Xavier, em Seropédica, RJ. As espécies utilizadas foram: Eucalyptus urophylla "Eucalipto" (Myrtaceae), Melia azedarach L. "Cinamomo" (Meliaceae), Lophantera lactescens Ducke, "Lanterneira" (Malpighiaceae), Pinus elliottii "Pinus" (Pinaceae) e Inga marginata "Inga" (Mimosaceae). A madeira de Eucalipto é proveniente de um plantio homogêneo de 7 anos e a de Pinus, de um plantio de 11 anos. As demais madeiras vieram de árvores de mesma idade, pois se tratam de plantios homogêneos, mas de idade desconhecida.

Foram cortadas seis árvores de cada espécie, sendo estas demarcadas com giz para posterior processamento, adotando-se o seguinte ordenamento: a cada $150 \mathrm{~cm}$ marcava-se uma tora, do mesmo modo, em seqüência, marcava-se um torete de $40 \mathrm{~cm}$, e assim por diante a até uma circunferência préestabelecida de 25 a $35 \mathrm{~cm}$.

A sistemática de coleta descrita anteriormente visa minimizar o efeito da variação da densidade da madeira na avaliação das propriedades mecânicas antes e após o intemperismo, pois, toda a possível variação ao longo do tronco estará representada nos toretes e toras, que foram obtidos em seqüência ao longo dos fustes. 
Foram montados dois campos de apodrecimento, um dentro de uma floresta secundária e outro em um pasto composto por gramíneas. Seis toras, em cada ambiente, foram enterradas distantes $1 \mathrm{~m}$ uma da outra, verticalmente a uma profundidade de $30 \mathrm{~cm}$, de forma que $120 \mathrm{~cm}$ ficassem expostos ao ar.

Os toretes de $40 \mathrm{~cm}$ serviram para a confecção dos corpos-de-prova da madeira não exposta às intempéries, realizada logo após o abate da árvore. Nas toras de $150 \mathrm{~cm}$ foram retirados, da parte mediana da peça, os corpos-de-prova da madeira exposta às intempéries, nos dois ambientes, depois de 12 meses de exposição nos dois campos de apodrecimento.

A madeira não intemperizada, oriunda dos toretes de $40 \mathrm{~cm}$, foi levada para a marcenaria logo após o corte das árvores, para a confecção dos corpos-de-prova (condição I). Cada sequiência de dados é relativa a um dos ambientes, ou seja, campo a céu aberto (condição II) e campo de dentro da mata (condição III).

Foram confeccionados 240 corpos-de-prova retirados da região do cerne, de dimensão nominal de $2 \times 2 \times 30 \mathrm{~cm}$, para avaliação dos módulos de elasticidade (MOE) e de ruptura (MOR) na flexão estática, sendo 120 oriundos dos toretes de $40 \mathrm{~cm}$ não expostos às intempéries e 120 das toras de $150 \mathrm{~cm}$ expostas às intempéries. Destes últimos, 60 foram oriundos das toras expostas no campo de apodrecimento a céu aberto (condição II) e 60, expostas no campo de apodrecimento de dentro da mata (condição III), o que culminou em 12 corpos-de-prova para cada uma das cinco espécies, no respectivo ambiente. Para realização dos ensaios, os corpos-de-prova foram dispostos na máquina de ensaio mecânico,, de forma que a carga fosse aplicada perpendicularmente aos anéis de crescimento.

Para avaliação da resistência à compressão paralela às fibras, foram confeccionados 960 corpos-de-prova também retirados da madeira do cerne, de dimensão nominal de $2 \times 2 \times 3 \mathrm{~cm}$, sendo 480 oriundos dos toretes de $40 \mathrm{~cm}$ não intemperizados e 480 das toras expostas às intempéries. Estes últimos (240) foram procedentes de cada um dos dois campos de apodrecimento, o que resultou em 48 corpos-de-prova para cada uma das cinco espécies, no respectivo ambiente.

Foi adotado um índice de deterioração adaptado de Lepage (1970) (Quadro 1), para avaliação da decomposição dos corpos-de-prova, onde, através do dano neles causados, notas foram atribuídas à geração do índice médio de deterioração.

Após a confecção dos corpos-de-prova, estes foram aclimatados em câmara climática com $20^{\circ} \mathrm{C}$ e $65 \%$ de umidade relativa até atingirem peso constante; nessa condição, o teor de umidade final ficou em torno de $12 \%$. Antes dos ensaios mecânicos, os corpos-de-prova foram pesados e medidos com o auxílio de um paquímetro digital, procedendo-se ao cálculo da densidade aparente pelo método esteriométrico. O delineamento experimental foi o inteiramente casualizado. As médias foram analisadas pelo teste $\mathrm{T}$ a $5 \%$ de significância entre condições e pelo teste de Tukey a 5\% de significância, entre espécies. Os ensaios mecânicos foram realizados em máquina universal de ensaio da marca EMIC, utilizando a norma MB 26 (NBR 6230).

\section{RESULTADOS E DISCUSSÃO}

Os valores médios das temperaturas, máxima e mínima nos campos de apodrecimento foram: 27 e $20^{\circ} \mathrm{C}$ para dentro da mata e 32 e $20^{\circ} \mathrm{C}$ para o campo a céu aberto. Oliveira et al. (1986) afirmaram que as condições de temperatura, dentre outros fatores, são importantes na determinação dos microrganismos aptos a colonizar a madeira e decompô-la e têm marcante influência na velocidade da decomposição. Temperaturas entre 5 e 65 ${ }^{\circ} \mathrm{C}$ permitem o desenvolvimento desses microrganismos, entretanto são poucos os que crescem acima de $35 \mathrm{ou}$ $40^{\circ} \mathrm{C}$. No entanto, como diferentes espécies de organismos apresentam diferentes tolerâncias e pontos ótimos de temperaturas, esse fator pode influenciar intensamente a composição de espécies das populações que colonizaram o material nos diferentes estágios seriais da degradação.

Quadro 1 - Classificação do nível de degradação dos corpos-de-prova

Table 1-Classification of degradation level of the wood specimens

\begin{tabular}{lcc}
\hline Estado de sanidade & Nota & Índice de deterioração \\
\hline Sadio, nenhum dano visível. & 0 & 100 \\
Ataque leve ou superficial de fungos, térmitas ou coleobrocas. & 1 & 90 \\
Ataque evidente mas moderado de fungos, térmitas ou coleobrocas. & 2 & 70 \\
Apodrecimento intenso ou ataque interno de térmitas. & 3 & 40 \\
Deterioração intensa, com desfibramento evidente. & 4 & 0 \\
\hline
\end{tabular}

Fonte: Lepage, 1970. 


\subsection{Degradação dos corpos-de-prova}

Os corpos-de-prova oriundos de dentro da mata apresentaram-se mais degradados em comparação com os de fora da mata. Esse fato é absolutamente compreensível em função das características ecológicas inerentes ao ambiente florestal, características essas que favorecem a diversidade e a atuação dos organismos decompositores da madeira.

As amostras da madeira de $M$. azedarach e $L$. lactescens não apresentaram danos evidentes a olho nu, a não ser pela presença de alguns fungos manchadores, sendo registrado um índice médio de deterioração de 100, para ambos os ambientes (Quadro 2). Esse índice não ratifica que microrganismos e processos abióticos não tenham atuado na degradação dessas madeiras de forma intensa, sendo sua interpretação relacionada com danos aparentes, evidentes e macroscópicos.

Os corpos-de-prova mais atacados, oriundos de toras expostas fora e dentro da mata, segundo o índice de deterioração médio foram: E. urophylla, I. marginata e $P$. elliotti, sendo registrado um índice médio de 99 , 98 e 91, provenientes do campo a céu aberto, e 61, 73 e 86 provenientes do campo de dentro da mata, respectivamente (Quadro 2).

Embora seja conhecido que a madeira de Pinus apresente baixa durabilidade, foi registrado que os corposde-prova da madeira de Eucalipto apresentaram-se mais degradados. Comparando-se com os de Pinus, isso provavelmente pode ser explicado pela idade da árvore que foi coletada a madeira, sendo de 7 anos para Eucalipto e 11 anos para Pinus e pela baixa resistência a térmitas dessa espécie de Eucalipto.

Quadro 2 - Índice médio de deterioração dos corpos-deprova oriundos de toras expostas no campo de apodrecimento de fora da mata (condição II) e expostas no campo de apodrecimento de dentro da mata (condição III). Seropédica, 2005

Table 2 - Average decay index of wood specimens originated from wood exposed to decaying field outside the forest (condition II) and exposed to decaying field within the forest (condition III). Seropédica, 2005

\begin{tabular}{lcc}
\hline \multicolumn{1}{c}{ Madeira } & Condição II & Condição III \\
\hline I. marginata & 98 & 73 \\
L. lactescens & 100 & 100 \\
P. elliottii & 91 & 86 \\
M. azedarach & 100 & 100 \\
E. urophylla & 99 & 61 \\
\hline
\end{tabular}

Paes e Vital (2000), estudando a resistência natural da madeira de cinco espécies de Eucalipto a cupins subterrâneos em testes de laboratório, concluíram que E. urophylla foi a espécie que apresentou menor resistência, entre as avaliadas, nas posições internas do tronco, região de onde foram confeccionados os corpos-de-prova para este estudo.

Silva et al. (2004), avaliando a influência da idade na resistência natural da madeira de Eucalyptus grandis ao ataque de cupim de madeira seca, Cryptotermes brevis, demonstraram que, quanto mais jovem a madeira, maior a suscetibilidade ao ataque do cupim, e que a madeira de 10 anos, mais jovem entre as avaliadas, não apresentou diferença significativa no desgaste pelos térmitas, em comparação com a testemunha, Pinus elliottii.

\subsection{Propriedades físicas e mecânicas}

\subsubsection{Densidade aparente e resistência à compressão paralela às fibras}

\subsubsection{Densidade aparente dos corpos-de-prova destinados à compressão}

A redução porcentual média da densidade foi mais acentuada nas madeiras que foram expostas dentro da mata, sendo os maiores valores registrados para as madeiras de E. urophylla e $P$. elliottii, com redução de 14\% em ambas (Quadro 4), seguidas de M. azedarach e I. marginata, registrando valores de 8 e $5 \%$, respectivamente (Quadro 4). Venson et al. (2000), ao estudarem as propriedades mecânicas da madeira de Pinus taeda submetido à ação de agentes biodegradadores, por um período de exposição de seis meses dentro de um povoamento da mesma espécie, verificaram redução de 4,3\% na densidade. A madeira de L. lactecens não apresentou redução na densidade, em os ambos ambientes. Os valores médios da densidade das madeiras de E. urophylla, P. elliottii e M. azedarach, nas amostragens relativas ao campo de dentro da mata, diferiram significativamente pelo teste t a $5 \%$ de significância, entre as condições (Quadro 4).

Nas madeiras submetidas ao campo de apodrecimento a céu aberto, foi registrada uma redução de $1 \%$ nos valores de suas densidades, com exceção das de I. marginata e L. lactescens, que não denotaram 
redução na densidade, embora todas não tenham diferido estatisticamente antes e depois do intemperismo (Quadro 3). Essa variação não necessariamente está ligada a degradação biótica ou abiótica. Por se tratar de reduções baixas, estas poderiam estar relacionadas com a variação da amostragem, feita no fuste das árvores. No entanto, os corpos-de-prova procedentes de dentro da mata apresentaram sinais intensos da degradação causada por fungos e insetos, o que explica a maior redução da densidade na madeira submetida a esse ambiente.

\subsubsection{Resistência à compressão paralela às fibras}

Para as madeiras de E. urophylla e I. marginata, foi registrada uma redução significativa de $11 \%$ nos valores médios da resistência à compressão, no campo a céu aberto (condição II) (Quadro 5). No campo de dentro da mata (condição III), ocorreram reduções significativas nas madeiras de E. urophylla, P. elliottii e I. marginata, com porcentuais de 42, 28 e 20, respectivamente (Quadro 6).

Venson et al. (2000), ao estudarem a atuação de agentes biodegradadores na madeira de Pinus taeda, dentro de um povoamento homogêneo da mesma espécie, registraram uma redução porcentual de $14,7 \%$ nos esforços para a compressão, num período de seis meses de exposição às intempéries.

Quadro 3 - Densidade aparente média dos corpos-de-prova ( \pm desvio padrão), em $\mathrm{g} / \mathrm{cm}^{3}$, e redução percentual de madeiras de cinco espécies florestais que não foram exposta a intempéries (Condição I) e exposta em campo aberto (Condição II) por 12 meses. Seropédica, 2005

Table 3-Mean apparent density of wood specimens ( \pm standard deviation), in $\mathrm{g} / \mathrm{cm}^{3}$, and percentage of reduction of wood from five forest species that were not exposed to atmospheric conditions (Condition I) and exposed to open field (Condition II) for 12 months. Seropédica, 2005

\begin{tabular}{lcccc}
\hline \multicolumn{1}{c}{ Madeira } & \multicolumn{2}{c}{ Condição } & $\begin{array}{c}\text { Redução } \\
(\%)\end{array}$ \\
\cline { 2 - 4 } & I & II & 0 \\
I. marginata & $0,75 \pm 0,04$ a A & $0,75 \pm 0,04$ a A & 0 \\
L. lactescens & $0,75 \pm 0,04$ a A & $0,75 \pm 0,03$ a A & 0 \\
P. elliottii & $0,50 \pm 0,06$ c A & $0,49 \pm 0,06$ c A & 1 \\
M. azedarach & $0,45 \pm 0,03$ d A & $0,44 \pm 0,01$ d A & 1 \\
E. urophylla & $0,57 \pm 0,04$ b A & $0,56 \pm 0,04$ b A & 1 \\
\hline
\end{tabular}

Médias seguidas da mesma letra minúscula na coluna (Tukey $\mathrm{P}<$ 0,05 ) e maiúscula na linha (“t” $\mathrm{P}<0,05$ ) não diferem,significativamente, por ambos os testes.
Quadro 4-Densidade aparente média dos corpos-de-prova ( \pm desvio-padrão),emg/ $\mathrm{cm}^{3}$, ereduçãoporcentual demadeiras de cinco espécies florestais que não foram exposta a intempéries (Condição I) e expostas dentro da mata (Condição III), por 12 meses. Seropédica, 2005

Table 4-Mean apparent density of wood specimens ( \pm standard deviation), in $\mathrm{g} / \mathrm{cm}^{3}$, and percentage of reduction of wood fromfive forest species that were not exposed to atmospheric conditions (ConditionI) and frominside the forest (Condition III), for 12 months. Seropédica, 2005

\begin{tabular}{|c|c|c|c|}
\hline \multirow[t]{2}{*}{ Madeira } & \multicolumn{2}{|c|}{ Condição } & \multirow{2}{*}{$\begin{array}{c}\text { Redução } \\
(\%)\end{array}$} \\
\hline & I & II & \\
\hline I. marginata & $0,76 \pm 0,04$ a $A$ & $0,72 \pm 0,07$ a $\mathrm{B}$ & 5 \\
\hline L. lactescens & $0,74 \pm 0,03$ a $A$ & $0,74 \pm 0,04$ a $A$ & 0 \\
\hline P. elliottii & $0,49 \pm 0,04 \mathrm{c} \mathrm{A}$ & $0,42 \pm 0,04$ c $B$ & 14 \\
\hline M. azedarach & $0,49 \pm 0,04 \mathrm{c} \mathrm{A}$ & $0,45 \pm 0,03$ c $\mathrm{B}$ & 8 \\
\hline E. urophylla & $0,57 \pm 0,03 \mathrm{~b} \mathrm{~A}$ & $0,49 \pm 0,04 \mathrm{~b} \mathrm{~B}$ & 14 \\
\hline
\end{tabular}

Médias seguidas da mesma letra minúscula na coluna (Tukey $\mathrm{P}<$ 0,05 ) e maiúscula na linha ("t" $\mathrm{P}<0,05$ ) não diferem, significativamente, por ambos os testes.

Quadro 5 - Valores médios de compressão paralela às fibras ( \pm desvio-padrão), em kgf/ $\mathrm{cm}^{2}$, eperdaporcentual damadeira de cinco espécies florestais que não foram expostas a intempéries (Condição I) e expostas em campo aberto (Condição II) por 12 meses. Seropédica, 2005

Table 5-Mean values of parallel compression to the fibers ( \pm standard deviation), in $\mathrm{kgf} / \mathrm{cm}^{2}$ and percentage of loss of wood from five forest species that were not exposed to atmospheric conditions (Condition I) and exposed to open field (Condition II) for 12 months. Seropédica, 2005

\begin{tabular}{lcccc}
\hline Madeira & \multicolumn{3}{c}{ Condição } & $\begin{array}{c}\text { Redução } \\
(\%)\end{array}$ \\
\cline { 2 - 3 } & I & II & \\
\hline I. marginata & $473 \pm 42$ ab A & $418 \pm 48$ b B & 11 \\
L. lactescens & $509 \pm 77$ a A & $499 \pm 65$ a A & 2 \\
$P$. elliottii & $347 \pm 54$ c A & $341 \pm 70$ c A & 2 \\
M. azedarach & $332 \pm 33$ c A & $330 \pm 28$ c A & 0,6 \\
E. urophylla & $446 \pm 35$ b A & $395 \pm 49$ b B & 11 \\
\hline
\end{tabular}

Médias seguidas da mesma letra minúscula na coluna (Tukey $\mathrm{P}<$ 0,05 ) e maiúscula na linha ("t"' $\mathrm{P}<0,05$ ) não diferem, significativamente, por ambos os testes.

\subsubsection{Densidade aparente, módulo de ruptura (MOR) e elasticidade (MOE) na flexão estática}

\subsubsection{Densidade aparente dos corpos-de-prova destinados à flexão estática}

A redução porcentual da densidade, no campo a céu aberto (condição II), atingiu valores semelhantes aos dos corpos-de-prova destinados à compressão, sendo que as médias também permaneceram iguais

R. Árvore, Viçosa-MG, v.31, n.1, p.93-101, 2007 
estatisticamente, após o intemperismo, quando aplicado o teste t (Quadro 7). Apenas para a madeira de $P$. elliottii foi registrada uma redução maior nesse parâmetro, em comparação com os corpos-de-prova destinados à compressão. Embora não significativa, essa redução pode ter sido ocasionada pelo tamanho do corpo-deprova destinado à flexão estática, sendo bem maior que o destinado à compressão, abrangendo, dessa forma, maior área mensurável e, conseqüentemente, maior representação dos processos de degradação, sejam bióticos ou abióticos.

A redução porcentual da densidade, após o intemperismo, nos corpos-de-prova oriundos do campo de apodrecimento de dentro da mata (condição III) apresentou valores semelhantes aos dos corpos-deprova destinados à compressão, sendo que as maiores perdas também foram registradas nas madeiras de $P$. elliottii e E.urophylla, ambas com reduções de $14 \%$ cada, seguidas por I. marginata $6 \%$ e M. azedarach $4 \%$. As médias da densidade, dessas madeiras apresentaram-se diferentes ao serem submetidas à análise pelo teste t, após o intemperismo (Quadro 8).

\subsubsection{Módulo de ruptura (MOR) na flexão estática}

O processo de degradação ocasionou reduções porcentuais diferenciadas em cada ambiente, no módulo de ruptura. Com relação às madeiras expostas no campo a céu aberto (condição II), as que tiveram registros significativos estatisticamente foram as madeiras de E. urophylla e P. elliottii, com reduções de 20 e $19 \%$, respectivamente, sendo as médias dessa variável e dessas madeiras, antes e depois do intemperismo, diferentes quando comparadas pelo teste $\mathrm{t}$ a $5 \%$ de significância (Quadro 9).

Para as madeiras expostas no campo de dentro da mata (condição III), apenas L. lactescens não apresentou redução significativa, pelo teste t, no módulo de ruptura, após o intemperismo, sendo registrada uma redução de 5\% (Quadro 10). As demais madeiras, $E$. urophylla, P. elliottii, I.marginata e M. azedarach, perderam, respectivamente, 52, 49, 36 e $22 \%$ nos valores de MOR após o intemperismo, sendo diferentes pelo teste t (Quadro 10).

Embora a madeira de E. urophylla não seja a segunda mais densa, ela se apresentou como a segunda mais resistente quanto ao módulo de ruptura, nas duas amostragens, com relação à madeira não exposta às intempéries (Condição I) (Quadros 9 e 10); isso se torna possível devido a questões da anatomia da respectiva madeira, ou seja, questões como a orientação da grã podem conferir a madeira maior resistência à ruptura, em comparação com uma madeira mais densa, no caso I. marginata. Essa propriedade deixou de ser expressa quando a respectiva madeira ficou exposta às intempéries durante um ano, nos dois ambientes, pois a maior redução porcentual da resistência à ruptura, causada pela maior suscetibilidade aos agentes degradadores, expressa por essa madeira, tornou esta a terceira mais resistente no campo de apodrecimento a céu aberto (Quadro 9) e a quarta mais resistente no campo de apodrecimento de dentro da mata (Quadro 10).

\subsubsection{Módulo de elasticidade na flexão estática}

As maiores reduções porcentuais no módulo de elasticidade, após o intemperismo, foram registradas em $P$. elliottii e E urophylla, observando-se valores porcentuais de 21 e 20 , respectivamente, para a comparação com a condição II (Quadro 11) e de 35 e 38, respectivamente, para a amostragem que foi comparada com a condição III (Quadro 12), sendo essas reduções significativas pelo teste $\mathrm{t}$ a $5 \%$ de significância, pois as médias antes e depois da exposição às intempéries são diferentes estatisticamente (Quadros 11 e 12).

Quadro 6 - Valores médios de compressão paralela às fibras ( \pm desvio-padrão)em kgf $/ \mathrm{cm}^{2}$, eperda porcentualdamadeira de cinco espécies florestais que não foram expostas a intempéries (Condição I) e expostas dentro da mata (Condição III) por 12 meses. Seropédica, 2005

Table 6-Mean values of parallel compression to the fibers ( \pm standard deviation) in $\mathrm{kgf} / \mathrm{cm}^{2}$, and percentage of loss of wood from five forest species, that were not exposed atmospheric conditions (Condition I) and from inside the forest (Condition III) for 12 months. Seropédica, 2005

\begin{tabular}{lcccc}
\hline Madeira & \multicolumn{3}{c}{ Condição } & $\begin{array}{c}\text { Redução } \\
(\%)\end{array}$ \\
\cline { 2 - 4 } & I & II & (\% \\
\hline I. marginata & $446 \pm 61$ ab A & $357 \pm 86$ b B & 20 \\
L. lactescens & $480 \pm 56$ a A & $464 \pm 49$ a A & 3 \\
P. elliottii & $321 \pm 25$ c A & $232 \pm 34$ c B & 28 \\
M. azedarach & $351 \pm 32$ & c A & $325 \pm 33$ b A & 7 \\
E. urophylla & $412 \pm 42$ & b A & $239 \pm 72$ c B & 42 \\
\hline
\end{tabular}

Médias seguidas da mesma letra minúscula na coluna (Tukey $\mathrm{P}<$ 0,05 ) e maiúscula na linha ("t" $\mathrm{P}<0,05$ ) não diferem, significativamente, por ambos os testes. 
Quadro 7 - Densidade aparente média dos corpos-de-prova ( \pm desvio-padrão), em g/cm³ , e redução percentual, da madeira de cinco espécies florestais que não foram expostas à intempéries (Condição I) e exposta em campo aberto (Condição II) por 12 meses. Seropédica, 2005

Table 7-Mean apparent density of wood specimens ( \pm standard deviation), in $\mathrm{g} / \mathrm{cm}^{3}$, and percentage of reduction of wood from forest species that were not exposed to atmospheric conditions (Condition I) and exposed to open field (Condition II) for 12 months. Seropédica, 2005

\begin{tabular}{lcccc}
\hline Madeira & \multicolumn{3}{c}{ Condição } & $\begin{array}{c}\text { Redução } \\
(\%)\end{array}$ \\
\cline { 2 - 4 } & I & II & 1 \\
I. marginata & $0,76 \pm 0,05$ a A & $0,75 \pm 0,04$ a A & 1 \\
L. lactescens & $0,76 \pm 0,04$ a A & $0,76 \pm 0,03$ a A & 0 \\
P. elliottii & $0,52 \pm 0,06$ c A & $0,50 \pm 0,07$ b A & 4 \\
M. azedarach & $0,45 \pm 0,02$ d A & $0,45 \pm 0,02$ c A & 0 \\
E. urophylla & $0,55 \pm 0,02$ b A & $0,55 \pm 0,03$ b A & 0 \\
\hline
\end{tabular}

Médias seguidas da mesma letra minúscula na coluna (Tukey $\mathrm{P}<$ 0,05 ) e maiúscula na linha (“t”" $\mathrm{P}<0,05$ ) não diferem, significativamente, por ambos os testes.

Quadro 8 - Densidade aparente média dos corpos-de-prova ( \pm desvio-padrão), em g/ $\mathrm{cm}^{3}$, e redução porcentual de madeiras de cinco espécies florestais que não foram expostas a intempéries (Condição I) e expostas dentro da mata (Condição III) por 12 meses. Seropédica, 2005

Table 8-Mean apparent density of wood specimens ( \pm standard deviation), in $\mathrm{g} / \mathrm{cm}^{3}$, and percentage of reduction of wood from five forest species that were not exposed to atmospheric conditions (Condition I) and from inside the forest (Condition III) for 12 months. Seropédica, 2005

\begin{tabular}{lcccc}
\hline Madeira & \multicolumn{3}{c}{ Condição } & $\begin{array}{c}\text { Redução } \\
(\%)\end{array}$ \\
\cline { 2 - 5 } & I & II & \\
\cline { 2 - 4 } I. marginata & $0,77 \pm 0,04$ a A & $0,72 \pm 0,05$ a B & 6 \\
L. lactescens & $0,74 \pm 0,03$ a A & $0,74 \pm 0,07$ a A & 0 \\
P. elliottii & $0,49 \pm 0,02$ c A & $0,42 \pm 0,06$ c B & 14 \\
M. azedarach & $0,48 \pm 0,02$ c A & $0,46 \pm 0,02$ bc B & 4 \\
E. urophylla & $0,57 \pm 0,02$ b A & $0,49 \pm 0,03$ b B & 14 \\
\hline
\end{tabular}

Médias seguidas da mesma letra minúscula na coluna (Tukey $\mathrm{P}<$ 0,05 ) e maiúscula na linha ("t" $\mathrm{P}<0,05$ ) não diferem, significativamente, por ambos os testes.

A madeira de E. urophylla apresentou comportamento semelhante ao módulo de ruptura, pois, não sendo a segunda mais densa, apresentou o segundo maior módulo de elasticidade. O processo de degradação no campo de apodrecimento de dentro da mata e a maior suscetibilidade da madeira de E. urophylla aos organismos xilófagos a transferiram da posição de segunda mais elástica para a quarta (Quadro 12). Essa diminuição após o intemperismo é justificada pela maior perda porcentual dessa propriedade, nesse ambiente, e entre as madeiras avaliadas. Esse comportamento não foi notado no campo de apodrecimento a céu aberto, como no módulo de ruptura, o que indica que a diminuição no módulo de elasticidade foi menos influenciada pelos fatores que atuaram no campo a céu aberto, em comparação com o módulo de ruptura, que teve sua resistência diminuída, nesse ambiente, da segunda para a terceira maior resistência, após o intemperismo.

Quadro 9 - Valores médios do módulo de ruptura (MOR), em kgf $/ \mathrm{cm}^{2}$ ( \pm desvio-padrão), e redução porcentual da madeira de cinco espécies florestais que não foram expostas a intempéries (Condição I) e expostas em campo aberto (Condição II) por 12 meses. Seropédica, 2005

Table 9 - Mean values of modulus of rupture (MOR), in kgf/ $\mathrm{cm}^{2},( \pm$ standard deviation) and percentage of reduction of wood from five forest species that were not exposed to atmospheric conditions (Condition I) and exposed to open field (Condition II) for 12 months. Seropédica, 2005

\begin{tabular}{lccc}
\hline Madeira & \multicolumn{2}{c}{ Condição } & $\begin{array}{c}\text { Redução } \\
\text { I }\end{array}$ \\
\cline { 2 - 3 } I. marginata & $699 \pm 145$ bc A & $657 \pm 67$ b A & 6 \\
L. lactescens & $952 \pm 221$ a A & $908 \pm 148$ a A & 6 \\
P. elliottii & $607 \pm 99$ bc A & $489 \pm 131$ c B & 19 \\
M. azedarach & $576 \pm 106$ c A & $570 \pm 64$ bc A & 1 \\
E. urophylla & $756 \pm 49$ b A & $600 \pm 70$ bc B & 20 \\
\hline
\end{tabular}

Médias seguidas da mesma letra minúscula na coluna (Tukey $\mathrm{P}<$ $0,05)$ e maiúscula na linha (“t”' $\mathrm{P}<0,05$ ) não diferem, significativamente, por ambos os testes.

Quadro 10 - Valores médios do módulo de ruptura (MOR), em kgf/ $/ \mathrm{cm}^{2}$ ( \pm desvio-padrão) e redução porcentual da madeira de cinco espécies florestais que não foram expostas a intempéries (Condição I) e expostas dentro da mata (Condição III) por 12 meses. Seropédica, 2005

Table 10 - Mean values of Modulus of rupture (MOR), in $\mathrm{kgf} / \mathrm{cm}^{2}$, ( \pm standard deviation) and percentage of reduction of wood from five forest species that were not exposed to atmospheric conditions (Condition I) and from inside the forest (Condition III) for 12 months. Seropédica, 2005

\begin{tabular}{|c|c|c|c|}
\hline \multirow[t]{2}{*}{ Madeira } & \multicolumn{2}{|c|}{ Condição } & \multirow{2}{*}{$\begin{array}{c}\text { Redução } \\
(\%)\end{array}$} \\
\hline & $\mathrm{I}$ & II & \\
\hline I. marginata & $706 \pm 163 \mathrm{ab} \mathrm{A}$ & $454 \pm 174$ bc B & 36 \\
\hline L. lactescens & $849 \pm 200$ a $A$ & $811 \pm 186$ a $A$ & 5 \\
\hline P. elliottii & $530 \pm 80 \mathrm{c} \mathrm{A}$ & $272 \pm 92 \mathrm{~d} B$ & 49 \\
\hline M. azedarach & $638 \pm 120$ bc $A$ & $499 \pm 59 \mathrm{~b} \mathrm{~B}$ & 22 \\
\hline E. urophylla & $710 \pm 53 \mathrm{ab} A$ & $338 \pm 72 \mathrm{~cd} \mathrm{~B}$ & 52 \\
\hline
\end{tabular}

Médias seguidas da mesma letra minúscula na coluna (Tukey $\mathrm{P}<$ 0,05 ) e maiúscula na linha (“t” $\mathrm{P}<0,05$ ) não diferem, significativamente, por ambos os testes. 
Quadro 11 - Valores médios do Módulo de Elasticidade (MOE), em kgf $/ \mathrm{cm}^{2}$ ( \pm desvio-padrão), e redução porcentual da madeira de cinco espécies florestais que não foram expostas a intempéries (Condição I) e expostas em campo aberto (Condição II) por 12 meses. Seropédica, 2005

Table 11 - Mean values of Modulus of Elasticity (MOE), in $\mathrm{kgf} / \mathrm{cm}^{2}$, ( \pm standard deviation) and percentage of reduction of wood from five forest species that were not exposed to atmospheric conditions (Condition I) and exposed to open field (Condition II) for 12 months. Seropédica, 2005

\begin{tabular}{lccc}
\hline Madeira & \multicolumn{2}{c}{ Condição } & $\begin{array}{c}\text { Redução } \\
(\%)\end{array}$ \\
\cline { 2 - 4 } & I & II & 6 \\
I. marginata & $50.712 \pm 11.394 \mathrm{cA}$ & $47.715 \pm 11.479 \mathrm{bcA}$ & 6 \\
L. lactescens & $79.299 \pm 15.935 \mathrm{aA}$ & $76.309 \pm 7.209 \mathrm{aA}$ & 4 \\
P. elliottii & $49.684 \pm 9.664 \mathrm{cA}$ & $39.211 \pm 10.436 \mathrm{cB}$ & 21 \\
M. azedarach & $46.633 \pm 12.041 \mathrm{cA}$ & $46.131 \pm 6.507 \mathrm{bcA}$ & 1 \\
E. urophylla & $65.775 \pm 7.217 \mathrm{bA}$ & $52.845 \pm 7.364 \mathrm{bB}$ & 20 \\
\hline
\end{tabular}

Médias seguidas da mesma letra minúscula na coluna (Tukey $\mathrm{P}<$ 0,05 ) e maiúscula na linha ("t" $\mathrm{P}<0,05$ ) não diferem,significativamente, por ambos os testes.

Quadro 12 - Valores médios do Módulo de Elasticidade (MOE), em kgf $/ \mathrm{cm}^{2}$ ( \pm desvio-padrão), e redução porcentual da madeira de cinco espécies florestais que não foram expostas a intempéries (Condição I) e expostas dentro da mata (Condição III) por 12 meses. Seropédica, 2005

Table 12 - Mean values of Modulus of Elasticity (MOE), in $\mathrm{kgf} / \mathrm{cm}^{2},( \pm$ standard deviation) and percentage of reduction of wood from five forest species that were not exposed to atmospheric conditions (Condition I) and from inside the forest (Condition III) for 12 months. Seropédica, 2005

\begin{tabular}{lccc}
\hline Madeira & \multicolumn{2}{c}{ Condição } & $\begin{array}{c}\text { Redução } \\
\text { II }\end{array}$ \\
\cline { 2 - 3 } & \multicolumn{1}{c}{ I } & II \\
\hline I. marginata & $52.576 \pm 14.906 \mathrm{bcA}$ & $41.457 \pm 11.925 \mathrm{bA}$ & 21 \\
L. lactescens & $72.035 \pm 13.467 \mathrm{aA}$ & $69.613 \pm 13.004 \mathrm{aA}$ & 3 \\
P. elliottii & $39.831 \pm 5.330 \mathrm{cA}$ & $26.001 \pm 9.380 \mathrm{cB}$ & 35 \\
M. azedarach & $47.856 \pm 4.757 \mathrm{cA}$ & $45.049 \pm 5.629 \mathrm{bA}$ & 6 \\
E. urophylla & $60.042 \pm 6.608 \mathrm{bA}$ & $37.451 \pm 5.606 \mathrm{bcB}$ & 38 \\
\hline
\end{tabular}

Médias seguidas da mesma letra minúscula na coluna (Tukey $\mathrm{P}<$ 0,05 ) e maiúscula na linha (" $t$ " $\mathrm{P}<0,05$ ) não diferem, significativamente, por ambos os testes.

Nas madeiras de I. marginata, L. lactescens e $M$. azedarach, foram registradas reduções porcentuais de 6; 4 e 1\% para a comparação com a condição II (Quadro 11), e para a comparação com a Condição III registraramse valores de 21; 3 e 6\% (Quadro 12). Em ambas as amostragens, as médias antes e depois do intemperismo demonstraram-se iguais estatisticamente pelo teste t a 5\% de significância. Embora em I. marginata tenha sido registrada uma redução de $21 \%$ no módulo de elasticidade, essa redução, embora não significativa estatisticamente, corroborou o índice de deterioração médio (73) obtido pelos corpos-de-prova oriundos do campo de dentro da mata, os quais tiveram essa redução porcentual. Apesar de a madeira $P$. elliotti ter um índice de deterioração maior (86), isso poderia sugerir uma menor atuação dos processos de degradação, mas devese entender que essas notas são relativas a avaliações visuais, desconsiderando a atuação dos processos que não são perceptíveis a olho nu. Portanto, isso poderia explicar a maior redução no módulo de elasticidade da madeira de $P$. elliotti, em comparação com a madeira de I. marginata, além da diferença entre as densidades dessas madeiras.

\section{CONCLUSÕES}

- As madeiras mais suscetíveis aos organismos xilófagos foram E. urophylla e I. marginata e as menos atacadas, L. lactescens e M. azedarach.

- O ambiente influenciou a ocorrência dos organismos xilófagos, bem como as reduções das propriedades físicas e mecânicas.

- A densidade das madeiras de L. lactescens, $M$. azedarach, E. urophylla, I. marginata e Pinus elliotti não sofreu influência do intemperismo no campo de apodrecimento a céu aberto, em 12 meses de exposição.

- A densidade, a resistência à compressão e os módulos de elasticidade (MOE) e ruptura (MOR) na flexão estática da madeira de L. lactecens não foram afetados significativamente pelo intemperismo nos dois ambientes.

- A densidade das madeiras de M. azedarach, E. urophylla, I. marginata e Pinus elliotti foi afetada pelo intemperismo no campo de dentro da mata.

- A madeira de L. lactescens e I. marginata apresentaram maior resistência à compressão antes e depois do intemperismo, nos dois ambientes.

- As reduções na resistência à compressão, demonstraram-se mais acentuadas nas madeiras expostas no campo de apodrecimento de dentro da mata, sendo os maiores porcentuais registrados na madeira de $E$. urophylla.

- As maiores reduções nos módulos de ruptura foram registrados no campo de apodrecimento de dentro da mata por estas madeiras: E. urophylla, P. elliottii e I. marginata, nessa seqüência. 
- O intemperismo interfere, de forma mais acentuada, nas propriedades da madeira de E. urophylla, nos dois ambientes.

- As maiores reduções no módulo de elasticidade na flexão estática ocorrem nas madeiras das espécies de E. urophylla e P. elliottii, nos dois campos de apodrecimento.

\section{REFERÊNCIAS}

APRILE, F. M.; DELITTI, W. B. C.; BIANCHINI JR., L. Aspectos cinéticos da degradação de laminados de madeira em ambientes aquático e terrestre. Revista Brasileira de Biologia, v.59, n.3, p.485-492, 1999.

\section{ASSOCIAÇÃO BRASILEIRA DE NORMAS} TÉCNICAS - ABNT. MB 26 (NBR 6230), Ensaios físicos e mecânicos de madeiras. Rio de janeiro: 1940. 9p.

LEPAGE, E. S. Método sugerido pela IUFRO para ensaios de campo com estacas de madeira. Preservação de Madeiras, v. 1 , p.205-216,1970.
OLIVEIRA, A.M.F. et al. Agentes destruidores da madeira. In: LEPAGE, E. S. (Coord.) Manual de preservação de madeiras. São Paulo: IPT, 1986. v.1 p.99-279.

PAES, J. B.: VITAL, B. R. Resistência natural da madeira de cinco espécies de eucalipto a cupins subterrâneos, em teste de laboratório. Revista Árvore, v.24, n.1, p.97-104, 2000.

SILVA, J. C.; CABALLEIRA LOPEZ, A. G.; OLIVEIRA, J. T. S. Influência da idade na resistência natural da madeira de Eucalyptus grandis W. Hill ex. Maiden ao ataque de cupim de madeira seca (Cryptotermes brevis). Revista Árvore, v.28, n.4, p.583-587, 2004.

SWIFT, M. J.; HEAL, D. W.; ANDERSON, J. M. Studies in ecology-decomposition in terrestrial and aquatic ecosystems. Oxford: Blackwell, 1979. p.54-94.

VENSON, I.; MATOS, J. L. M.; SOUZA, N. J. Propriedades físicas e mecânicas de Pinus taeda L. submetido à ação de agentes biodegradadores. Floresta, v.30, p.195, 2000. Edição Especial. 
